\title{
A MOMENT THEOREM
}

\author{
WILLIAM A. VEECH
}

Let $G$ be a separable, locally compact, topological abelian group. We denote by $C_{c}=C_{c}(G)$ the space of continuous functions with compact support on $G$ and by $C_{c}^{+}$the set of nonnegative real-valued functions in $C_{c}$, excluding the one which is identically zero. $\mathfrak{N}^{+}$is taken to be the class of nonnegative, locally finite Borel measures on $G$, and the topology on $\mathfrak{N T}^{+}$is that of weak* convergence on compact sets of $G$. To say that a function $g \geqq 0$ belongs to $\mathfrak{M}^{+}$is to say that it is locally integrable with respect to Haar measure $d x$ and therefore the density of $g d x \in \mathfrak{T}^{+}$. With this convention we will consider $G^{+}$, the set of positive continuous characters on $G$, to be a subset of $\mathrm{NC}^{+}$with the induced topology. So far as we know, the following theorem is new, even for $G$ the real line or integers.

Theorem 1. Let $f \geqq 0$ be a function on $G$ which has the property that for each $\phi \in C_{c}^{+}$there exists $\mu \in \mathfrak{N}^{+}$with $\phi * \mu=f$. Then there exists a nonnegative Borel measure $\nu$ on $G^{+}$such that

$$
f(x)=\int_{G^{+}} g_{0}(x) \nu\left(d g_{0}\right) \quad(x \in G) .
$$

A function satisfying the hypotheses of the theorem will be called divisible.

REMARK 1. One can prove that if in addition the group $G$ is divisible (for each $x \in G$ and integer $n>0$ there exists $y \in G$ with $n y=x$ ), then $\nu$ is unique. Otherwise this need not be so. For example, when $G$ $=$ integers, $G^{+}=$real line, then $\nu_{1}(d x)=\exp \left(-x^{2}\right) d x$ and $\nu_{2}(d x)$ $=\left(\exp \left(-x^{2}\right)\right)(1-\sin (2 \pi x)) d x$ have the same transforms (1) (see $[4$, p. 22]).

REMARK 2. The converse to Theorem 1 is also true. Given $\phi \in C_{c}^{+}$, define $\hat{\phi}\left(g_{0}\right)=\int_{G} g_{0}(-x) \phi(x) d x$, and let $\nu_{0}\left(d g_{0}\right)=\nu\left(d g_{0}\right) / \hat{\phi}\left(g_{0}\right)$. One checks easily that $g(x)=\int_{G^{+}} g_{0}(x) \nu_{0}\left(d g_{0}\right)$ satisfies $\phi * g=f$.

To establish the representation (1) we will apply a device of Furstenberg together with the Choquet-Bishop-de Leeuw theorem (in its weakest form) to some rather simple observations. Motivation for the theorem itself has come from our study of [1].

Lemma 1. For any $f \geqq 0$ on $G$ and $\phi \in C_{c}^{+}$, the set

Received by the editors February 3, 1967. 


$$
B_{\phi}=\left\{\mu \in \Re^{+} \mid \phi * \mu=f\right\}
$$

is compact.

Proof. Since $\phi \not \equiv 0$, there exists a $\delta>0$ such that $U=\{x \mid \phi(x)>\delta\}$ is nonempty. Thus it is clear for any $\mu \in B_{\phi}$ and $x \in G$ that $\mu(x-U)$ $\leqq f(x) / \delta$, and therefore the measures in $B_{\phi}$ are locally uniformly bounded. It follows that $B_{\phi}$ has compact closure in $\mathfrak{T}^{+}$. If $\mu=\lim _{n} \mu_{n}, \mu_{n} \in B_{\phi}$, then $\phi * \mu=\lim _{n} \phi * \mu_{n}=f$, and $\mu \in B_{\phi} . B_{\phi}$ is closed and therefore compact, and the lemma is proved.

Lemma 2. Fix $\phi \in C_{c}^{+}$. If $\left\{f_{n}\right\}$ is a net of functions on $G$ such that for each $n$ the equation $\phi * \nu=f_{n}$ has a solution $\nu=\nu_{n} \in \mathfrak{N}^{+}$, and if $\lim _{n} f_{n}$ $=\mu$ exists, then there exists $\nu \in \Re^{+}$with $\phi * \nu=\mu$.

Proof. Let $\delta>0$ and $U$ be as in the proof of Lemma 1. There exists a compact subset $U_{0}$ of $U$ with nonempty interior and a compact symmetric neighborhood $W$ of $e$ (the identity in $G$ ) such that $W+U_{0} \subseteq U$. Choose $x \in G$. The assumption $\lim _{n} f_{n}=\mu$ guarantees that there is an index $n_{0}$ such that if $n>n_{0}$, then

$$
\int_{x+W} f_{n}(y) d y \leqq \mu(x+W)+1 .
$$

Letting $|W|$ be the Haar measure of $W$ there exists $w=w_{n} \in W$ with

$$
f_{n}(x+w) \leqq(\mu(x+W)+1) /|W|=\alpha .
$$

We conclude as in Lemma 1 that

$$
\nu_{n}(x+w-U) \leqq \alpha / \delta
$$

and therefore, since $-U_{0} \subseteq w-U$,

$$
\nu_{n}\left(x-U_{0}\right) \leqq \alpha / \delta .
$$

The net $\left\{\nu_{n}\right\}$ is locally eventually uniformly bounded, and so it has a convergent subnet $\left\{\nu_{n_{k}}\right\}$. If $\nu$ is the limit of this subnet, we have $\phi * \nu=\lim _{k} \phi * \nu_{n_{k}}=\mu$, and the lemma is proved.

REMARK 3. Since $\phi * \nu=\mu, \mu$ has a density $f$, and our argument shows that $f(x)=\lim _{k} f_{n_{k}}(x)$ for each $x$. Since this is true for any convergent subnet, it must be that $\lim _{n} f_{n}(x)=f(x), x \in G$.

Lemma 3. Let $f$ be divisible. For each $\phi \in C_{c}^{+}$there exists a divisible function $g$ such that $\phi * g=f$.

Proof. If $\Psi=\left(\psi_{1}, \cdots, \psi_{n}\right)$ is an $n$-tuple from $C_{c}^{+}$, we denote by $A_{\phi}=A_{\phi}(\Psi)$ the subset of $B_{\phi}$ consisting of those $\mu$ for which the equations 


$$
\psi_{i} * \nu_{i}=\mu \quad(i=1, \cdots, n)
$$

have solutions $\nu_{1}, \cdots, \nu_{n} \in \mathfrak{N}^{+}$. By Lemma $2, A_{\phi}$ is closed, and we claim $A_{\phi} \neq \varnothing$. To see this, set $\psi=\psi_{1} * \cdots * \psi_{n} * \phi$, and note that $\psi \in$ $C_{c}^{+}$. Since $f$ is divisible, $B_{\psi} \neq \varnothing$. Select $\nu \in B_{\psi}$, and define

$$
\nu_{i}=\psi_{1} * \psi_{2} * \cdots * \hat{\psi}_{i} * \cdots * \psi_{n} * \nu, \quad \mu=\psi_{1} * \cdots * \psi_{n} * \nu
$$

where ${ }^{\wedge}$ denotes omission. Clearly $\mu \in B_{\phi}$ and $\psi_{i} * \nu_{i}=\mu$ for $i=1, \cdots n$. Thus $\mu \in A_{\phi}$, and $A_{\phi} \neq \varnothing$.

From the argument just given we see that the sets $A_{\phi}(\Psi)$, where $\Psi$ ranges over the finite subsets of $C_{c}^{+}$, have the finite intersection property. By the compactness of $B_{\phi}$ there exists a measure $\mu \in B_{\phi}$ common to all of these sets, and such a measure is obviously given by a density which is a divisible function. The lemma is proved.

We denote by $\Delta=\Delta(G)$ the cone of divisible functions on $G . \Delta$ is closed in $\mathfrak{T}^{+}$by Lemma 2 , and if $\phi \in C_{c}^{+}$, we have $\phi * \Delta \subseteq \Delta$. Therefore, by Lemma $3, \phi * \Delta=\Delta, \phi \in C_{c}^{+}$.

An extremal of $\Delta$ is a function $g$ such that if $g=g_{1}+g_{2}$ with $g_{1}, g_{2} \in \Delta$, then both $g_{1}$ and $g_{2}$ are proportional to $g$. The following argument is due to Furstenberg ([1, especially Theorem 12.2]); it shows that the extremals of $\Delta$ are proportional to elements of $G^{+}$. Let $g \in \Delta$ be an extremal, and fix $\phi \in C_{c}^{+}$. By Lemma 3 there exists $g_{1} \in \Delta$ with $\phi * g_{1}=g$. This equation expresses $g$ as a "linear combination" of elements of $\Delta$, and therefore the translates of $g_{1}$ by negatives of elements in the support of $\phi$ must be proportional to $g$. If $e$ is in the support of $\phi$, then $g_{1}$ is itself proportional to $g$. Thus, by taking $\phi$ to have arbitrarily large (compact) support, we find all translates of $g$ proportional to $g$. It follows that $g=g(e) g_{0}, g_{0} \in G^{+}$. Furstenberg's argument is complete.

To prove Theorem 1 we first determine (using the separability of $G$ ) a "cap" of $\Delta$ which contains $f$. This is done by choosing a function $h>0$, continuous on $G$, such that $\int_{G} f(x) h(x) d x \leqq 1$. Then $D$ $=\left\{g \in \Delta \mid \int_{G} g(x) h(x) d x \leqq 1\right)$ is compact, convex and its extreme points are extremals of $\Delta([1],[3])$.

Denote by $D_{e}$ the set of extreme points of $D$. Since the elements of $D_{e}$ are either positive multiples of characters or else 0 , and since by Remark $3, \mathfrak{T}^{+}$limits in $D$ are pointwise limits, $D_{e}$ is closed. In this situation an argument based on the Krein-Milman theorem guarantees the existence of a measure $\nu_{0} \geqq 0$ on $D_{e}$ such that

$$
f=\int_{D_{c}} \tilde{g}_{0} \nu_{0}\left(d \tilde{g}_{0}\right) \text {. }
$$

Unless $f \equiv 0$ we may assume $\nu_{0}$ to be concentrated on $D_{s}-\{0\}$, and therefore 


$$
\nu\left(d g_{0}\right)=\tilde{g}_{0}(e) \nu_{0}\left(d \tilde{g}_{0}\right) \quad\left(g_{0}=\tilde{g}_{0} / \tilde{g}_{0}(e)\right)
$$

defines a measure on $G^{+}$. For $\nu$ (1) follows from (3), and Theorem 1 is proved.

Uniqueness in (1) is proved for divisible $G$ by substituting $g_{0}^{2}(x)$ for $g_{0}(x)$ in (1) and noting that the entire function arising is the same for any solution $\nu$. Letting $z$ be purely imaginary the mapping $g_{0} \rightarrow g_{0}^{z}$ is a one-one homomorphism from $G^{+}$in to $G^{\wedge}$, the character group of $G$. In this situation the uniqueness theorem for the Fourier transform implies the uniqueness of $\nu$.

Note. Karlin and Loewner [2] have given a "cone theoretic" characterization of the bilateral Laplace transforms of nonnegative measures on the line. It would be interesting to establish directly the equivalence of their conditions with ours. I thank the referee for pointing this paper out to me.

\section{BIBLIOGRAPHY}

1. H. Furstenberg, Translation invariant cones of functions on semisimple Lie groups, Bull. Amer. Math. Soc. 71 (1965), 271-326.

2. S. Karlin and C. Loewner, "On some classes of functions associated with exponential polynomials" in Studies in mathematical analysis and related topics, Stanford Univ. Press, Stanford, Calif., 1962, pp. 175-182.

3. R. R. Phelps, Lectures on Choquet's Theorem, Van Nostrand, Princeton, N. J., 1966.

4. J. A. Shohat and J. D. Tamarkin, The problem of moments, Math. Surveys, Vol. 1, Amer. Math. Soc., Providence, R. I., 1943.

University of California, Berkeley 